\title{
SAZONALIDADE DA PRODUÇÃO E CARACTERÍSTICAS DO LÁTEX DE CLONES DE SERINGUEIRA EM LAVRAS, MG ( $\left.{ }^{1}\right)$
}

\author{
ALESSANDRO CARLOS MESQUITA $\left({ }^{2 *}\right)$; LUIZ EDSON MOTA DE OLIVEIRA $\left({ }^{3}\right)$; \\ PAULO ARAQUÉM RAMOS CAIRO $\left({ }^{4}\right)$; ANTÔNIO AUGUSTO MOREIRA VIANA $\left({ }^{5}\right)$
}

\begin{abstract}
RESUMO
A despeito de ser o berço das espécies do gênero Hevea, o Brasil contribuiu, em 2001, com apenas $1,5 \%$ da produção mundial de 7,132 milhões de toneladas e consumiu em torno de 3,5\% de um total de 7,03 milhões de toneladas da demanda mundial de borracha. Os clones mais produtivos são os orientais RRIM 600, PB 235 e PR 255, com produção em torno de $1.500 \mathrm{~kg}$ de borracha seca/ha/ano, o que evidencia sua adaptação a vários padrões climáticos. O presente trabalho teve como objetivo avaliar o comportamento produtivo de clones de seringueira [(Hevea brasiliensis (Willd.ex. Adr. de Juss.) Muell. Arg.], associado a fatores climáticos sazonais e fisiológicos sobre a produção de látex em Lavras (MG). O trabalho foi desenvolvido na área experimental do Departamento de Biologia, Setor de Fisiologia Vegetal, da Universidade Federal de Lavras, Minas Gerais, Brasil, durante o período de junho de 2001 a julho de 2003. Os fatores hídricos e nutricionais das plantas foram adequados ao desempenho produtivo, sendo o clone RRIM 600 amplamente superior aos demais, além de apresentar correlação com os fatores ambientais durante o período avaliado. Os fatores nutricionais do látex e o índice de obstrução indicaram correlação significativa com a produção.
\end{abstract}

Palavras-chave: correlação entre caracteres, látex, produção, seringueira.

\begin{abstract}
SAZONAL PRODUCTION AND LATEX CHARACTERISTICS IN RUBBER TREE (HEVEA BRASILIENSIS MUELL. ARG.) CLONES IN LAVRAS, STATE OF MINAS GERAIS, BRAZIL

In spite of being the cradle of the species of the genus Hevea, Brazil contributed, in 2001, with only $1.5 \%$ of the world production of 7.132 million tons and it consumed around $3.5 \%$ of a total of 7.03 million tons of the world demand. The most productive clones are the orientals RRIM 600, PB 235 and PR 255, with production around 1,500 kg of dry rubber/ha/year. The cultivation of those clones in different regions in Brazil shows adaptation to several climatic patterns. The aim of this work was to evaluate the productive behavior of clones of rubber tree, associated to seasonal climatic and physiologic factors in relation to the latex production in Lavras, State of Minas Gerais, Brazil. The research was conducted in the experimental area of Biology Department of Federal University of Lavras, sector of Plant Physiology, between June 2001 and July 2003. The plant water relations and nutritional factors were appropriate to the productive period. The productivity of clone RRIM 600 was higher than the others and presented positive correlation to the environmental factors during the experimental period. The nutritional factors of the latex and the obstruction index showed significant correlation to the production.
\end{abstract}

Key words: correlation caracters, production, rubber plant.

( $\left.{ }^{1}\right)$ Recebido para publicação em 2 de fevereiro e aceito em 15 de julho de 2006.

$\left(^{2}\right)$ Departamento de Biologia. Universidade Federal de Lavras. PD/CNPq. E-mail: mesquita@ufla.br. ${ }^{*}$ Autor correspondente.

$\left({ }^{3}\right)$ Departamento de Biologia. Universidade Federal de Lavras. E-mail: ledson@ufla.br

$\left({ }^{4}\right)$ Departamento de Fitotecnia e Zootecnia, Universidade Estadual do Sudoeste da Bahia. E-mail: pcairo@uol.com.br

$\left({ }^{5}\right)$ Mestrando em Fisiologia Vegetal. Universidade Federal de Lavras E-mail: gutoufla.@yahoo.com.br 


\section{INTRODUÇÃO}

Desde o início da domesticação da seringueira, há cerca de 120 anos, sua produtividade, em termos de produção de borracha, tem se elevado de $400 \mathrm{~kg} \mathrm{ha}^{-1} /$ ano para $3.000 \mathrm{~kg} \mathrm{ha}^{-1} / \mathrm{ano}$ (GonçALVES, 2002). Com área de plantio superior a 10 milhões de hectares no Brasil, a heveicultura extrapolou tradicionais áreas de cultivo - Amazônia Tropical Úmida, Mato Grosso e Bahia - abrangendo atualmente nove Estados brasileiros. Em Minas Gerais, as plantações estão concentradas nas regiões do Triângulo Mineiro e do Alto Paranaíba.

A despeito de ser o berço das espécies do gênero Hevea, o Brasil contribuiu, em 2001, com apenas $1,5 \%$ da produção mundial de 7,132 milhões de toneladas e consumiu em torno de $3,5 \%$ de um total de 7,03 milhões de toneladas da demanda mundial (GAMEIRO, 2003). Em virtude da grande demanda por borracha natural, além da tentativa de superação de problemas de origem fitossanitária, a heveicultura se expandiu para regiões cujas características ambientais são distintas de seu habitat. A seringueira tem bom desenvolvimento vegetativo e produtividades experimentais promissoras no sul de Minas Gerais, onde o inverno é marcado por baixas temperaturas e reduzido índice pluviométrico. Entretanto, ainda são necessários estudos que comprovem a viabilidade do cultivo de seringueira nessa região, devido à diversidade de condições edafoclimáticas.

Dentre os vários fatores que influenciam a produção de látex da seringueira, o estado hídrico da planta é um dos que se destaca. Resulta da interação de outros fatores, tais como demanda evaporativa da atmosfera, estado hídrico do solo, densidade de plantio, sistema de cultivo e processos fisiológicos (Abrams et al., 1992; Silva, 1999). Processos fisiológicos, tais como crescimento celular e fotossíntese, além da produtividade, são fortemente influenciados pelo potencial hídrico e seus componentes (CORRÊA et al., 1987 e SILVA, 1999).

Com base no conhecimento atual sobre o escoamento do látex, pesquisadores do Centro de Cooperação Internacional em Pesquisa Agronômica para o Desenvolvimento (CIRAD), em Montpelier (França), estabeleceram um diagnóstico do látex base em indicadores determinados por procedimentos simples, capazes de representar os aspectos mais importantes da dinâmica do escoamento e da regeneração, sendo admitidos como parâmetros fisiológicos do látex (Eschbach et al., 1984; PREVOT et al., 1986; ЈАСОв et al., 1988; ЈАСОв et al., 1989). Os parâmetros utilizados no diagnóstico do látex são: 1) sólidos totais; 2) teor de sacarose; 3) teor de tióis; 4) teor de magnésio; 5) teor de fósforo inorgânico; 6) $\mathrm{pH}$; 7) potencial redox; 8) índice de ruptura de lutóides.

O presente trabalho teve como objetivo avaliar a produção de borracha de clones de seringueira [Hevea brasiliensis (Willd. ex Adr. de Juss.) Muell. Arg.] e sua relação com fatores climáticos sazonais e fisiológicos do látex, em Lavras, MG.

\section{MATERIAL E MÉTODOS}

Este trabalho foi desenvolvido no período de junho de 2001 a junho de 2003, em plantios de seringueira com 12 anos de idade, localizados no Setor de Fisiologia Vegetal do Departamento de Biologia da UFLA, em Lavras (MG), situados a $918 \mathrm{~m}$ de altitude, latitude $21^{\circ} 14^{\prime} \mathrm{S}$ e longitude $45^{\circ} 00^{\prime} \mathrm{W}$ GRW. O clima da região caracteriza-se por duas estações bem definidas: seca e fria, de abril a setembro, e chuvosa e quente, de outubro a março. Durante o período experimental, foram monitoradas as condições ambientais (temperaturas máxima e mínima, precipitação pluvial, insolação e umidade relativa do ar) obtidas na Estação Climatológica localizada a cerca de 300 metros da área experimental.

De janeiro a maio de 2003, foi avaliado o potencial hídrico dos clones RRIM 600, Fx 2261 e GT 1. Os folíolos utilizados obedeceram sempre à mesma orientação cardeal em relação à radiação solar incidente, estando no estádio de desenvolvimento foliar D (completamente expandido), conforme descrição de Hallé et. al. (1978). Para a determinação do potencial hídrico utilizou-se uma bomba de pressão (bomba de Scholander), sendo o horário das avaliações entre $5 \mathrm{~h} 30$ e $6 \mathrm{~h} 30 \mathrm{~min}$.

A avaliação do estado nutricional do látex foi feita em amostras contendo $0,5 \mathrm{~g}$ de borracha seca triturada, às quais foram adicionados $6 \mathrm{~mL}$ de $\mathrm{HNO}_{3}$ e $\mathrm{HClO}_{4}$, na proporção de 2:1. Este material ficou em repouso por 24 horas, sendo depois colocado em bloco digestor, no qual a temperatura foi elevada até $160{ }^{\circ} \mathrm{C}$, e mantida até o volume ficar reduzido à metade. Em seguida, a temperatura foi elevada a $210^{\circ} \mathrm{C}$, até que fosse obtido um extrato incolor. O extrato foi resfriado à temperatura ambiente e, em seguida, foram adicionados $13 \mathrm{~mL}$ de água destilada, para a quantificação dos nutrientes: nitrogênio $(\mathrm{N})$, fósforo $(\mathrm{P})$, cálcio $(\mathrm{Ca})$ e magnésio $(\mathrm{Mg})$.

O sistema de sangria adotado foi o de meia espiral ( $1 / 2 \mathrm{~S})$, com duas sangrias por semana, espaçadas de 3 a 4 dias $(\mathrm{d} / 3$ e d/4), realizadas a 1,20 $\mathrm{m}$ da superfície do solo. Durante todo o período experimental não foram utilizados estimulantes para 
aumentar o fluxo de látex. Nos meses de agosto e setembro, a sangria foi suspensa, devido ao desfolhamento das plantas. Os resultados foram indicados em gramas de borracha seca por planta por sangria (g bs / planta/ sangria).

O índice de obstrução foi obtido pela equação:

$$
\text { I.O. }=\frac{\text { Volume de látex aos primeiros cinco minutos }}{\text { Volume final }} \times 100
$$

O delineamento utilizado foi o inteiramente casualizado, com três tratamentos e cinco repetições por tratamento e a análise estatística obtida pelo programa SANEST. O estudo da correlação simples entre os fatores e a produção de látex foi obtido utilizando-se também o programa SANEST, com cinco repetições por tratamento.

\section{RESULTADOS E DISCUSSÃO}

Na figura 1 observam-se as variações dos fatores ambientais ocorridas na cidade de Lavras (MG) durante o período de junho de 2001 a junho de 2003, condições climáticas distintas, especialmente em relação às temperaturas mínima e máxima e à precipitação pluvial. Entre junho e julho de 2001, a ausência de precipitação pluvial, a temperatura mínima de $12^{\circ} \mathrm{C}$ e a alta insolação caracterizaram esse período como seco e frio. A umidade relativa do ar se manteve constante durante esse período.
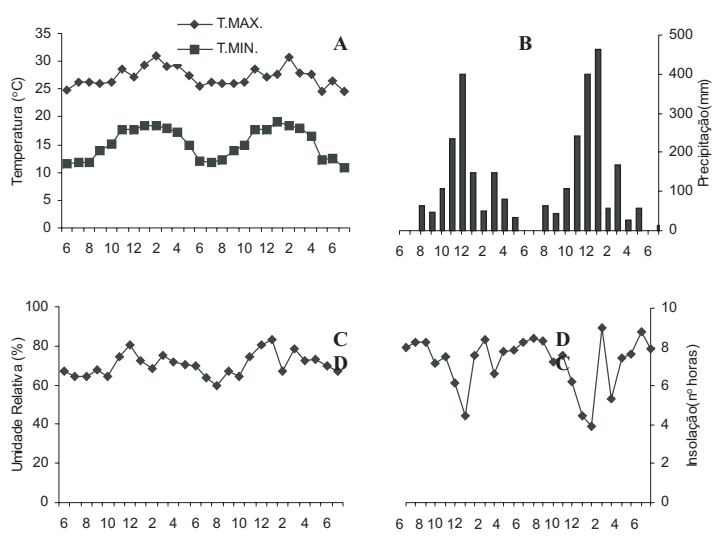

Mês/Ano

Mês/Ano

Figura 1. Média mensal das temperaturas máxima e mínima do ar (A), total mensal da precipitação (B) e total mensal da radiação global (C) e média mensal da umidade relativa do ar (D), durante o período experimental de junho de 2001 (6) e junho de 2003 (6). Fonte: Estação Meteorológica da UFLA, Lavras (MG).

No período que abrange agosto de 2001 a maio de 2002, ocorreu uma elevação nas temperaturas mínima e máxima, como também na precipitação pluvial, que atingiu seu máximo em dezembro de 2001.
Em agosto de 2001 e maio de 2002, os valores médios de precipitação pluvial foram similares, associados à elevação das temperaturas mínima e máxima, elevação da umidade relativa do ar e queda na intensidade luminosa. Em comparação com o mesmo período de 2001, em agosto de 2002 ocorreu apenas uma alteração na precipitação pluvial, devido à pequena incidência de chuva. Nos demais parâmetros avaliados, não houve diferença no comportamento climático nas duas estações. Os valores climatológicos indicados na figura 1 foram utilizados para o estudo da correlação entre temperatura máxima, temperatura mínima, precipitação pluvial, umidade relativa e luminosidade (Tabela 1), com os dados de produção dos três clones avaliados (RRIM 600, GT 1 e Fx 2261).

Tabela 1. Valores dos coeficientes de correlação linear simples entre produção de borracha de três clones de seringueira e cinco variáveis ambientais, em Lavras (MG)

\begin{tabular}{lccc}
\hline Parâmetros & \multicolumn{3}{c}{ Produção de borracha } \\
\cline { 2 - 4 } climatológicos & RRIM 600 & GT 1 & Fx 2261 \\
\hline Temperatura máxima & $0,29^{*}$ & 0,04 n.s. & 0,05 n.s. \\
Temperatura mínima & $0,12^{*}$ & $-0,51^{*}$ & $-0,51^{*}$ \\
Precipitação pluvial & $-0,06$ n.s. & $-0,92^{*}$ & $-0,92^{*}$ \\
Umidade relativa & $-0,001$ n.s. & $-0,85^{*}$ & $-0,85^{*}$ \\
Luminosidade & $0,23^{*}$ & $-0,16^{*}$ & 0,02 n.s. \\
\hline
\end{tabular}

* significativo a $5 \%$. n..s.: não siginficativo.

KshiRAgar (1999) relacionou a produção de látex com os fatores ambientais (temperaturas mínima e máxima, umidade relativa, insolação e precipitação pluvial) em volume de látex por sangria $(\mathrm{mL})$ em três clones de seringueira (RRIM 600, GT 1 e PR 107) e em uma planta de pé-franco. $O$ trabalho foi realizado na Índia e demonstrou um comportamento diferente do observado na região de Lavras (MG), entre os quais, o clone mais produtivo foi o GT $1(61,97 \mathrm{~mL})$, seguido pelo RRIM 600 (53,79 mL) e, com pior desempenho, o PR 107 e o clone sem enxertia produziu 95,95 mL. Além disso, foram observadas correlações positivas de $67 \%, 13 \%$ e $29 \%$, respectivamente, para os clones, e de $73 \%$ para o pé-franco, com relação aos fatores climáticos, durante o período de sangria.

No presente trabalho, as correlações lineares entre os parâmetros climatológicos e a produção de borracha diferiram dos valores obtidos por KSHIRAGAR (1999). De acordo com a tabela 1, nos clones GT 1 e Fx 2261 ocorreram correlações lineares semelhantes. Por outro lado, no clone RRIM 600 não houve correlação entre precipitação pluvial e umidade relativa com a produção e, ao contrário dos outros dois clones, verificou-se correlação positiva entre temperatura máxima, temperatura mínima e luminosidade ( $\mathrm{r}=0,29 ; 0,12$ e 0,23 respectivamente). 
Em estudos sobre correlações entre variáveis climáticas e a produção de látex realizados por RAO et al., (1998), observou-se uma correlação negativa para os valores máximos de temperatura, insolação, déficit de pressão de vapor e evapotranspiração, e somente a precipitação pluvial uma correlação positiva. Esses mesmos autores relatam que as condições ideais para o cultivo da seringueira são associadas com valores de temperatura máxima, mínima, insolação e precipitação pluvial de $30,4{ }^{\circ} \mathrm{C}$, $22,8^{\circ} \mathrm{C}, 5,9$ h e $72 \mathrm{~mm}$ respectivamente.

Os valores de potencial hídrico permaneceram praticamente inalterados durante os meses avaliados (março, abril e maio), não havendo diferença significativa entre as médias para os clones avaliados (em torno de $-0,4 \mathrm{MPa}$ ). Não foram mais realizadas avaliações em decorrência do fato das plantas perderem as folhas no período de baixa temperatura.

Resultados semelhantes foram obtidos por Oliveira (1999), em relação aos clones RRIM 600 e GT 1, quando, em julho e setembro, foram obtidos altos valores de potencial hídrico foliar (em torno de $-0,35$ MPa). Porém, de acordo com os resultados obtidos por essa pesquisadora, em dezembro, o potencial hídrico foliar foi significativamente diferente $(-0,15 \mathrm{MPa})$, devido ao fato de as precipitações terem sido mais constantes.

Períodos prolongados de estiagem que ocorrem durante o inverno na cidade de Lavras podem acarretar reduções expressivas de potencial hídrico foliar. Entretanto, a resistência estomática pode minimizar essas reduções de potencial hídrico, constituindo-se inclusive em uma das causas de variabilidade fotossintética entre clones de seringueira (Miguel, 2005).

$\mathrm{O}$ déficit de pressão de vapor (DPV) variou entre $-0,4$ e $-1,0 \mathrm{KPa}$ (dados não apresentados). Os valores de Kpa, por serem pouco expressivos, confirmam a inexistência de déficit hídrico durante o período de avaliação.

A produção de borracha seca nos três clones avaliados, durante o período de julho de 2001 a junho de 2003, revelou superioridade do clone RRIM 600, em relação aos demais clones, em todos os meses avaliados, com valor médio em torno de $41,0 \mathrm{~g}$ bs/ sangria, sendo esse valor semelhante aos obtidos pelos produtores no Brasil. Por esses dados, verifica-se que no clone RRIM 600 não houve redução no seu potencial produtivo quando submetido às condições climáticas de Lavras (MG). O clone GT 1 teve produção intermediária (21,0 g bs/sangria), correspondendo aproximadamente à metade da produção média obtida no clone RRIM 600. O clone Fx 2261 foi aquele com os menores valores médios para a produção de borracha (5,0 g bs/sangria) sendo este fato determinante para que esse clone não seja recomendado para plantios comerciais nessa localidade (Figura 2).

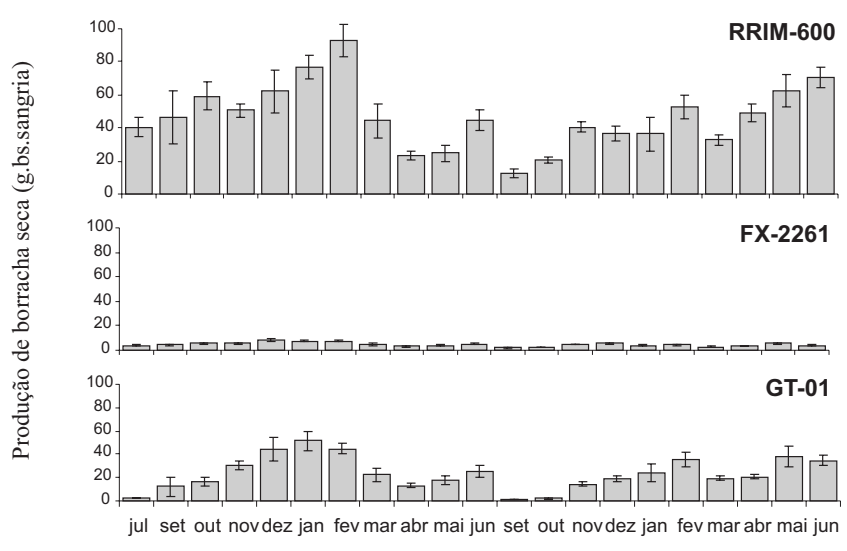

Mês/Ano(2001/02/03)

Figura 2. Produção de borracha seca das plantas de três clones de seringueira durante o período de julho/2001 a junho/2003, em Lavras (MG).

A produção de borracha do clone RRIM 600 em julho de 2001 foi semelhante à de setembro do mesmo ano, havendo, em seguida, um aumento na produção até fevereiro de 2002, quando foi obtida a maior produção de borracha seca por sangria. Vale ressaltar que, nesse período (Figura 1), houve elevação nas temperaturas mínima e máxima, como também na precipitação pluvial, favorecendo todos os processos envolvidos na incorporação, assimilação de carbono e produção de látex.

Em março de 2002, houve grande redução na produção de borracha, ainda menor em abril e se manteve constante em maio, com pequena elevação em junho. Observa-se que os valores obtidos em junho de 2002 foram semelhantes aos de abril do mesmo ano e que esse comportamento produtivo foi coerente com o obtido em julho de 2001.

É importante ressaltar que a produção de borracha tende a aumentar ano a ano e é crescente do primeiro até o quinto ano de avaliação quando então se estabiliza; entretanto, com a mudança de painel, mesmo depois de cinco anos de exploração pode haver decréscimo na produção. Vale também destacar que ainda não houve a mudança de painel nas plantas avaliadas nem a aplicação de estimulante.

Em julho e agosto de 2002 houve paralisação na sangria, devido ao reenfolhamento. Segundo RAO et al. (1998), o intenso crescimento da seringueira ocorre uma vez ao ano, com o surgimento de novos lançamentos após a queda natural das folhas. Durante esse período, entre o reenfolhamento e a maturidade dos novos folíolos, grande parcela da reserva de fotoassimilados estará sendo canalizada para renovação da folhagem, floração e frutificação 
e, conseqüentemente, apenas uma pequena parcela estaria sendo disponibilizada para a síntese de látex. É por isso que se deve evitar a sangria durante esse período, para não comprometer o crescimento das plantas e as produções futuras. De acordo com aqueles autores, no reenfolhamento, as condições meteorológicas devem ser consideradas, por influenciarem a capacidade da seringueira de acumular matéria seca. Neste caso, a radiação, por ser a fonte primária de energia, influencia diretamente a atividade fotossintética necessária ao crescimento e à produção de látex.

OLIVEIRA (1999), que avaliou a produção dos clones RRIM 600 e GT 1, nesse mesmo plantio, em julho, agosto, novembro e dezembro, registrou valores de produção do clone RRIM 600 significativamente inferiores $(20,0 ; 5,0 ; 5,0$ e 20,0 g bs/sangria respectivamente) aos obtidos neste trabalho. $\mathrm{O}$ clone GT 1 teve um comportamento ainda inferior, ficando os seus dados de produção, nos meses avaliados, na faixa de 5,0 g bs/sangria. É possível que as diferenças entre os resultados do presente trabalho, em comparação com os de Oliveira (1999) sejam justificados por diferenças na idade das plantas. Contudo, o comportamento produtivo foi semelhante ao observado para os clones RRIM 600 e GT 1, demonstrando superioridade do primeiro sobre o segundo, em produção de borracha seca. LimA (1998) e LiMA et al., (2002), trabalhando com o clone RRIM 600, em 1998, também nesse mesmo plantio, verificaram diferenças na produção de borracha seca por planta, em avaliações realizadas em épocas distintas (junho e dezembro), o que confirma a influência dos fatores ambientais sobre a produção. Esses autores relataram que a produção média de borracha em junho - mês com menor umidade relativa - foi de 8,5 g bs/sangria, aumentando significativamente para $17,5 \mathrm{~g}$ bs/sangria, em dezembro. Esses dados são de magnitudes inferiores às dos obtidos no presente trabalho. Entretanto, os autores justificaram a baixa produção pelo fato de serem referentes ao primeiro ano de sangria que, normalmente, tem menores produções, e dentre os fatores climatológicos, a precipitação pluvial e a temperatura foram os principais responsáveis pela maior produção registrada em dezembro.

Pelos resultados da tabela 2, verifica-se a influência da variação sazonal na composição de alguns ativadores minerais no látex de três clones de seringueira, em novembro de 2001 e junho de 2002. Alterações na nutrição mineral podem influenciar a atividade de algumas enzimas (PRADO, 1979; JACOB et al., 1982). Segundo esses autores, alguns ativadores, como fosfatos, nitratos e tióis, podem estar presentes no citosol em concentrações suficientes para influenciar a atividade da invertase neutra, cuja ação sobre a clivagem da sacarose representa uma etapachave na biossíntese de látex.

Tabela 2. Análise mineral da borracha seca em três clones de seringueira, em Lavras (MG)

\begin{tabular}{lccccccccc}
\hline \multirow{2}{*}{ Clone } & \multicolumn{4}{c}{ Novembro/2001 } & \multicolumn{4}{c}{ Agosto/2002 } \\
\cline { 2 - 9 } & $\mathrm{N}$ & $\mathrm{P}$ & $\mathrm{Ca}$ & $\mathrm{Mg}$ & $\mathrm{N}$ & $\mathrm{P}$ & $\mathrm{Ca}$ & $\mathrm{Mg}$ \\
\hline & \multicolumn{1}{c}{$\mathrm{g} / \mathrm{kg}-1$} \\
RRIM 600 & 0,97 & 0,12 & 0,04 & 0,06 & 0,72 & 0,05 & 0,03 & 0,04 \\
FX 2261 & 0,76 & 0,14 & 0,04 & 0,11 & 0,95 & 0,07 & 0,07 & 0,03 \\
GT 1 & 1,08 & 0,11 & 0,04 & 0,09 & 0,78 & 0,06 & 0,04 & 0,06 \\
\hline
\end{tabular}

Observa-se que houve redução nos teores de P e Mg em agosto de 2002, período em que a produção de borracha diminuiu. Em linhas gerais, as relações entre as concentrações desses nutrientes e o comportamento produtivo foram descritas por JАСОВ et al. (1988; 1989). Segundo esses autores, o fósforo inorgânico corresponde entre 60\% e 80\% do fósforo do látex, o que reflete a intensidade das trocas energéticas e, principalmente, a utilização do isopentenilpirofosfato na polimerização da borracha. O magnésio atua como ativador de enzimas, como ATPases, fosfoenolpiruvato carboxilase e fosfofrutoquinase, podendo ser limitante em concentrações muito baixas.

Além dos parâmetros citados anteriormente, o índice de obstrução é outro indicador da produção de borracha em seringueira, quando não se aplica nenhum estimulante à planta, e é expresso pela razão entre o fluxo inicial de látex até os cinco primeiros minutos da sangria e o volume total do látex exsudado no fim da sangria.

O estresse, devido ao período seco, deve induzir certas mudanças bioquímicas que levam à menor instabilidade dos lutóides, ocasionando maior obstrução ao fluxo de látex e, conseqüentemente, menor produção (DEVAKUMAR et al., 1988).

De acordo com a tabela 3, o índice de obstrução no clone RRIM 600 manteve-se praticamente inalterado nas duas épocas avaliadas, diferente do ocorrido nos clones Fx 2261 e GT 1, salientando-se o fato de não ter havido redução nos valores de produção. Entretanto, a redução nos valores de índice de obstrução encontrados no clone GT 1 de novembro de 2001 para maio de 2002, refletiu no aumento da produção, devido ao fato de ter ocorrido maior fluxo de látex durante a realização da sangria, não somente nos cinco primeiros minutos, mas durante todo o tempo de sangria. 
Tabela 3. Valores do índice de obstrução de três clones de seringueira em duas epócas, em Lavras (MG)

\begin{tabular}{|c|c|c|c|c|}
\hline \multirow{2}{*}{ Clone } & \multicolumn{2}{|c|}{ Índice de obstrução } & \multicolumn{2}{|c|}{ Produção } \\
\hline & Nov./2001 & Maio/2002 & Nov./2001 & Maio/2002 \\
\hline & \multicolumn{2}{|c|}{$\bar{\gamma} \%$} & \multicolumn{2}{|c|}{$\overline{\mathrm{g} \text { bs/sangria }-}$} \\
\hline RRIM 600 & 11,76 & 11,49 & 50,60 & 19,5 \\
\hline Fx 2261 & 13,78 & - & 5,90 & 4,11 \\
\hline GT 1 & 29,67 & 3,62 & 30,70 & 17,99 \\
\hline
\end{tabular}

Segundo Gonçalves et al., (1988), os critérios de redução do ciclo de melhoramento, utilizados na obtenção de cultivares de seringueira até então desenvolvidos, incluem o emprego de técnicas de seleção precoce. Dentre elas, destacamse os métodos indiretos, que incluem o uso de características fisiológicas, como o índice de obstrução, além de caracteres bioquímicos como elementos constituintes do látex, como N, P, K e outros (Ho, 1973). Segundo esses autores, somente o índice de obstrução e o número de anéis dos vasos laticíferos têm mostrado correlações significativas com produção de árvores adultas.

Foi realizado também o estudo da correlação entre o índice de obstrução e a produção de látex nos três clones avaliados. O coeficiente de correlação linear obtido $\left(r=0,39^{*}\right)$ foi significantivo e demonstra haver uma correlação positiva entre o índice de obstrução com a produção, podendo ser utilizado como fator importante na seleção de materiais promissores. HO et al. (1973) utilizaram centenas de clones obtidos de pés-francos previamente selecionados e observaram que é possível inferir sobre a produção de árvores adultas de clones por meio do índice de obstrução das plantas jovens. Os mesmos autores relatam que a seleção precoce utilizando esse critério teve o grande mérito de reduzir em cerca de $50 \%$ o número de clones a serem testados em experimentos, o que significou uma considerável redução no tamanho populacional e também no ciclo do melhoramento.

\section{CONCLUSÕES}

1. Os fatores hídricos e nutricionais da seringueira não foram limitantes à produção de borracha nos materiais avaliados.

2. Dentre os clones avaliados, o RRIM 600 foi superior em relação aos demais quanto à produção de borracha e foi aquele cuja produção demonstrou melhor adaptabilidade às condições ambientais de Lavras.

\section{AGRADECIMENTO}

Os autores agradecem o apoio financeiro recebido pelo $\mathrm{CNPq}$.

\section{REFERÊNCIAS}

ABRAMS, M. D.; KLOEPPEL, B. D.; KUBISKE, M. E. Ecophysiological and morphological responses to shade and drought in two contrasting ecotype of Prunus serotina. Tree Physiology, Victoria, v. 10, n. 4, p. 343-355, June 1992.

CORRÊA, A. F. F.; RANZANI, G.; FERREIRA, L. G. R. Relações entre o déficit hídrico e alguns processo fisiológicos e bioquímicos em quatro clones de seringueira. Acta Amazônica, Manaus, v. 16/17, p. 3-12, 1987.

DEVAKUMAR, A. S.; GURURAJA RAO, G.; RAJAGOPAL, R.; SANJEEVA RAO, P.; GEORGE, M. J.; VIJAYAKUMAR, K. R.; SETHURAJ, M. R. Studies on soil-plant-atmosphere system in Hevea: II. Seasonal effects on water relations and yield. Indian Journal of Natural Rubber Research, Keerala, v. 1, n. 1, p. 4560, 1988 .

ESCHBACH, J.M.; ROUSSEL, D.; SYPE, H.V.; JACOB, J.L. Relationships between yield and clonal physiological characteristics of latex form Hevea brasiliensis. Physiologie Végétale v.22, n.3, p.295-304, 1984.

GAMEIRO, A. H. Importação e produção de borracha natural no Brasil. Cepea, 1992 a 2002. Disponível em: $<$ www.cepea.esalq.usp.br)>. Acesso em: 25 ago. 2003.

GONÇALVES, P. S.; CARDOSO, M.; BORTOLETTO, N. Redução do ciclo de melhoramento e seleção na obtenção de cultivares de seringueira. O Agronômico, Campinas, v. 40, n. 2, p. 112130, maio/ago, 1988.

GONÇALVES, P. S. Uma história de sucesso: a seringueira no Estado de São Paulo. O Agronômico, Campinas, v. 54, n. 1, p. 6-14, 2002.

HALLÉ, F.; OLDEMAN, R. A.; TOMLINSON, P. B. Tropical trees and forest. Berlim, Springer-Verlag, 1978. $441 \mathrm{p}$.

HO, C. Y.; NARAYANAN, R.; CHEN, K. T. Clonal nursey studies in Hevea: I. Nursey yields and associated structural characteristic and their variations. Journal of the Rubber Research Institute of Malaya, Kuala Lumpur, v. 4, n. 23, p. 305-316, 1973.

JACOB, J. L.; PREVOT, J. C.; D'AUZAR, J. Physiological activators of invertase from Hevea brasiliensis latex. Phytochemistry, Oxford, v. 21, n. 4, p. 887-893, Apr. 1982.

JACOB, J. L.; PREVOT, J. C. Yield limiting factors, latex physiological parameters, latex diagnosis and clonal typology. In: D’AUZAC, J.; JACOB, J. L.; CHRESTIN, H. Physiology of rubber tree latex. Boca Raton: CRC Press, 1989. p. 345-382. 
JACOB. J. L.; SERRES, J. C. Mise au point du diagnostic látex chez I'Hevea. Agritop, Montpellier, v. 12, n. 2, p. 97-115, 1988.

KSHIRSAGAR, P. J. S. The latex yield of Hevea in relation to climatic factors. O Agronômico, Campinas, v. 51, n. 2, p. 1-4, 1999.

LIMA, D. U. Avaliação sazonal da produção e do metabolismo do carbono e nitrogênio em plantas de seringueira (Hevea brasiliensis Muell Arg.), cultivadas em Lavras, Minas Gerais. 1998. Dissertação (Mestrado em Fisiologia Vegetal) Universidade Federal de Lavras, Lavras, MG.

LIMA, D. U.; OLIVEIRA, L. E. M.;SOARES, A. M.; DELÚ-FILHO, N. Avaliação sazonal da produção de borracha e da dinâmica de carboidratos solúveis em plantas de seringueira (Hevea brasiliensis Muell. Arg.) cultivadas em Lavras, Minas Gerais. Revista Árvore, Viçosa, v. 36, n. 3, p. 377-383, maio/jun. 2002.

MIGUEL, A.A. Comportamento fotossintético caracterização morfo-anatômica de folhas de clones de seringueira (Hevea spp) em Lavras-MG. 2005. 182 f. Tese (Doutorado em Fisiologia Vegetal) Universidade Federal de Lavras, Lavras, MG.

OLIVEIRA, D. P. Avaliação sazonal da produção de látex e atividade de invertase em dois clones de seringueira (Hevea brasiliensis Muell Arg.), cultivadas em Lavras, Minas Gerais. 1999. 71 f. Dissertação (Mestrado em Fisiologia Vegetal) Universidade Federal de Lavras, Lavras, MG.
PRADO, F. E.; SAMPIETRO, A. R.; VATTUONE, M. A. Ammonium heptamolybdate, an inhibitor of plant invertase. Phytochemistry, Oxford, v. 18, p. 1799-1802, 1979.

PREVOT, J.C.; JACOB, J.L.; LACROTTE, R.; VIDAL, A.; SERRES, E.; ESCHBACH, J.M.; GIGAULT,J. Physiological parameters of latex from Hevea brasiliensis. Their use in the study of laticiferous suystem. Typology of functioning production mechanism. Effect of estimulation. In: IRRDB Rubber Physiology and Exploitation Meeting, Haiman, p.136-157, 1986.

RAO, P. S.; SARASWATJUA,,C. K.; SETHURAJ, M. R. Studies on the relationship between yield and meteorological parameters of para rubber tree (Hevea brasiliensis). Agricultural and Forest Meteorology, v. 90, p. 235-245, 1998.

SILVA, D. Respostas fisiológicas de três gramíneas promissoras para revegetação ciliar de reservatórios hidrelétricos submetidas a diferentes regimes hídricos. 1999. 74 f. Dissertação (Mestrado) - Universidade Federal de Lavras, Lavras, MG. 